\title{
The relationship between the down-regulation of DNA-PKes or Ku70 and the chemosensitization in human cervical carcinoma cell line HeLa
}

\author{
XIAOYU TIAN ${ }^{1,2^{*}},{\text { GANG } \mathrm{CHEN}^{1 *}, \mathrm{HUI} \mathrm{XING}^{1} \text {, DANHUI WENG }}^{1}$, YUNHONG GUO ${ }^{2}$ and DING MA ${ }^{1}$ \\ ${ }^{1}$ Cancer Biology Research Center, Tongji Hospital, Tongji Medical School, Huazhong University of Science \\ and Technology, Wuhan, Hubei 430030; ${ }^{2}$ Department of Obstetrics and Gynecology, First Affiliated \\ Hospital of Henan University of Science and Technology, Luoyang, Henan 471003, P.R. China
}

Received March 8, 2007; Accepted June 20, 2007

\begin{abstract}
The aim of this study was to clarify the function of non-homologous end-joining (NHEJ) in tumorigenesis and chemoresistance, and to explore the potential of DNA-PK as a target of reversal of chemoresistance and enhancing the sensitivity of cells to chemotherapeutic agents. Plasmid vectors pSIREN-Ku70shRNA and pSIREN-DNA-PKcsshRNA, which coded small interfering RNA of Ku70 and DNA-PKcs, were constructed and transfected into human cervical cancer cell line HeLa. The relationship between the down-regulation of Ku70 or DNA-PKcs and tumor cell proliferation and the sensitivity of cells to chemotherapeutic agents were analyzed. Down-regulation of Ku70 and DNAPKcs expression inhibited cell proliferation, and increased cell apoptosis in DDP-treated HeLa cells. DNA-PK might play an important role in drug resistance, and inhibition of the DNA-PK expression suppressed the growth of tumor cells and enhanced the sensitivity of cells to chemotherapeutic agents.
\end{abstract}

\section{Introduction}

Non-homologous end-joining (NHEJ) is the most important mechanism of DNA repair in mammalian cells. Competent

Correspondence to: Dr Ding Ma, Cancer Biology Research Center, Tongji Hospital, Tongji Medical College, Huazhong University of Science and Technology, 1095 Jiefang Ave., Wuhan, Hubei 430030, P.R. China

E-mail:dma@tjh.tjmu.edu.cn

${ }^{*}$ Contributed equally

Abbreviations: NHEJ, non-homologous end-joining; DNA-PK, DNA-dependent protein kinase; DNA-PKcs, catalytic subunit of DNA protein kinase; Ku70, regulatory subunit of DNA protein kinase; siRNA, small interfering RNA; DSBs, DNA double-strand breaks

Key words: tumor cell, DNA-PK, shRNA, transfection, drug sensitivity
NHEJ catalyses repair DNA double-strand breaks (DSBs) by ligating two free DNA ends with little or no homology. The DNA-dependent protein kinase (DNA-PK) holoenzyme, the main executive component of mammalian NHEJ, consists of a 470-kDa catalytic subunit (DNA-PKcs), a DNA-binding regulatory component known as $\mathrm{Ku}$ protein, and doublestranded DNA (dsDNA) with ends (1). DNA-PKcs is a nuclear serine/threonine protein kinase that comprises of a catalytic subunit, and the $\mathrm{Ku}$ subunits acting as the regulatory element (2). The DNA-PK activation, an essential step in the repair process, occurs once the kinase assembles at the site of DSB. The activation of DNA-PK may affect other downstream components involved in signal transduction of the damage event (3). This active DNA-PK complex may then recruit other factors including the MRE11-RAD50-NBS1 (MRN) complex, Artemis and the DNA ligase IV/XRCC4 complex (4-6), which are potentially involved in processing of the termini and completing the repair process. It has been proposed that DNA-PK is a molecular sensor for DNA damage that enhances the signal via phosphorylation of many downstream targets. Many studies demonstrated that the expression of DNA-PK was closely related to cellular radiosensitivity. More and more investigators pay close attention to the relationship between the down-regulation of DNA-PKcs or Ku70 and the chemosensitization. In order to investigate the function of DNA-PKcs and Ku70, we constructed RNAi expression plasmid vector pSIREN-Ku70shRNA and pSIREN-DNAPKcsshRNA which was designed against Ku70 and DNAPKcs mRNA, and transfected the plasmids into human cervical cancer cell line HeLa to test the silencing effect of target genes on cell proliferation, cell cycle, apoptosis, and to explore the potential of DNA-PK as a target for reversal of chemo-resistance.

\section{Materials and methods}

Cell culture. Human cervical cancer cell line HeLa was purchased from the American Type Culture Collection (ATCC, Manassas, VA) and cultured in RPMI-1640 (Gibco BRL) supplemented with $10 \%$ fetal bovine serum (Hangzhou Sijiqing Biological Engineering Materials Co. Ltd.). The cells were maintained at $37^{\circ} \mathrm{C}$ in a $5 \% \mathrm{CO}_{2}$ atmosphere. 
RNAi design and plasmid construction. Complementary siRNA oligonucleotides that targeted Ku70 or DNA-PKcs were designed according to "siRNA target finder and design tool' from 'hppt//www.ambion.com' (Fig. 1A). The oligonucleotides were synthesized (Invitrogen China, Shanghai), annealed and connected with pSIREN-DNR-DsRed-Express (BD Clontech) which expressed red fluorescent protein between the BamHI and EcoRI sites, and amplified in bacterium $E$. coli. All the recombinants were verified by DNA sequencing (Invitrogen China).

Transfection and drug interfering. The cells were seeded at a density of $5 \times 10^{5} / \mathrm{ml}$ on a 6 -well plate, according to the manufacturer's instructions, when the cell confluence reached $\sim 70-90 \%$, the mock vector pSIREN, and the recombinant plasmids pSIREN-Ku70shRNA1, 2 and pSIREN-DNAPKcsshRNA1, 2 were transfected into HeLa cells by Lipofectamine 2000 (Invitrogen). The transfection efficiency was determined by a fluorescence microscope.

For drug interfering, HeLa cells were exposed to the cisplatin (DDP), etoposide (VP-16) and topotecan (TPT) at different concentrations (DDP: 5, 10, 20, 40, 80, $160 \mu \mathrm{mol} / \mathrm{l}$; VP-16: 8, 16, 32, 64, 128, $256 \mu \mathrm{mol} / \mathrm{l}$; TPT: 10, 20, 40, 80, $160,320 \mu \mathrm{mol} / \mathrm{l})$. The cells were divided into four groups: the control, RNAi, chemotherapy (DDP, VP-16 or TPT) and RNAi + chemotherapy groups. Each group had 3 parallel wells.

$R T-P C R$. Cellular total RNA was extracted using Trizol (Invitrogen) and cDNA was synthesized with M-MLV reverse transcriptase (Promega) as recommended by the supplier. PCR reactions were performed using the primer pairs as follows: P1 5'-AAA GGA GGA CTC TGG AAA-3' and P2 5'-TGA TGT GGA TTT ATT G-3' to detect the 510-bp fragment of the Ku70; P1 5'-TTA TGC AGA AGC CCA GCT-3' and P2 5'-ATT ATG GAG TTT ACC ACG AC-3' for a 377-bp DNA-PKcs fragment, and P1 5'-ACG GAT TTG GTC GTA TTG GG-3' and P2 5'-TGA TTT TGG AGG GAT CTC GC-3' for a 230-bp fragment-coded internal control glyceraldehyde-3-phosphate dehydrogenase (GAPDH). Reaction cycle number was 30 cycles for Ku70 and DNAPKcs, 28 cycles for GAPDH. PCR was performed as follows: $95^{\circ} \mathrm{C}$ for $1 \mathrm{~min} ; 95^{\circ} \mathrm{C}$ for $30 \mathrm{sec}, 50^{\circ} \mathrm{C}$ for $45 \mathrm{sec}(\mathrm{Ku} 70)$, or $53^{\circ} \mathrm{C}$ for $45 \mathrm{sec}$ (DNA-PKcs), or $56^{\circ} \mathrm{C}$ for $30 \mathrm{sec}$ (GAPDH), and $72^{\circ} \mathrm{C}$ for $10 \mathrm{~min}$ for above mentioned cycles; the reactions were ended with $72^{\circ} \mathrm{C}$ for $10 \mathrm{~min}$.

Western blot analysis. Cells were lysed in RIPA buffer [50 $\mathrm{mmol} / \mathrm{l}$ Tris ( $\mathrm{pH} 8.0), 150 \mathrm{mmol} / \mathrm{l} \mathrm{NaCl}, 1 \% \mathrm{NP}-40,0.1 \%$ SDS, and $0.5 \%$ sodium deoxycholate]. The protein concentration of suspension was measured by the Coomassie Blue method. Equivalent amounts of whole-cell extracts $(80 \mu \mathrm{g})$ were separated in $10 \%$ polyacrylamide gel and transferred to Hybond-ECL nitrocellulose filter paper (Amersham). Filters were blocked in $25 \mathrm{mmol} / \mathrm{l}$ Tris (pH 8.0) containing $125 \mathrm{mmol} / 1 \mathrm{NaCl}, 0.1 \%$ Tween-20, and 5\% skim milk. Protein bands were probed with antibodies against Ku70 and DNA-PKcs at 1:300, and B-actin (1:1000), stayed overnight at $40^{\circ} \mathrm{C}$, and then with alkaline phosphatase-labeled secondary antibodies $(1: 500)$ at $37^{\circ} \mathrm{C}$ for $1 \mathrm{~h}$, the blots were developed by BCIP/NBT.
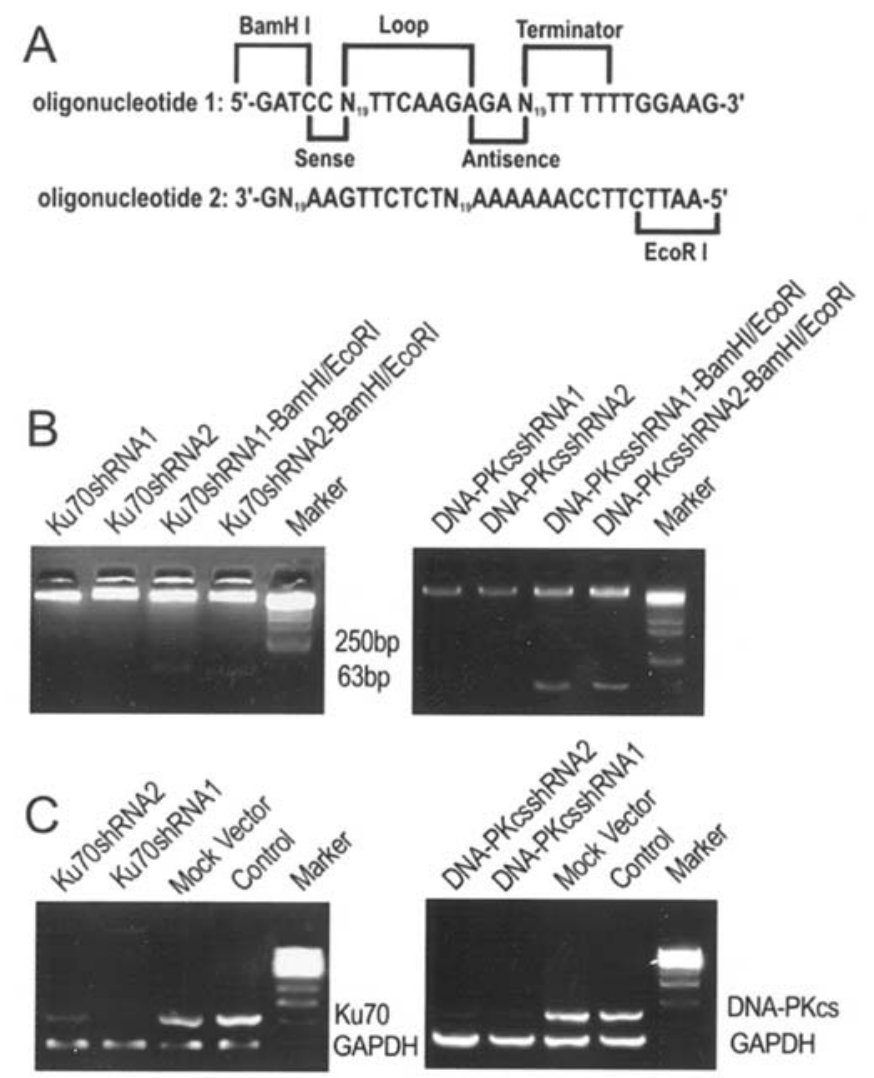

Figure 1. (A) DNA sequences that targeted the genes under study were inserted into the $\mathrm{N}_{19}$ both in forward and reverse. The complementary oligonucleotides were annealed to form a double-strand DNA which contained BamHI and EcoRI sites at both ends. Then the annealed DNA was ligated with BamHI/EcoRI-treated pSIREN-DNR-DsRed-Express. (B) BamHI/EcoRI digested recombinant plasmids. Each successful recombinant plasmid showed a 63-bp fragment in the agarose gel. All the plasmids were then sequenced to confirm no errors. (C) DNA-PKcs and Ku70 mRNA expression in transfected HeLa cells was detected by RT-PCR $48 \mathrm{~h}$ after transfection. DNA-PKcs and Ku70 mRNA were down-regulated dramatically after transfection with recombinant plasmids compared with mock plasmid-transfected and untransfected cells.

Cell immunohistochemistry. Sterilized coverslips were put into a 24 -well plate, cells were seeded at $10^{5}$ cells/well. When the confluence reached $\sim 50-70 \%$, the recombinant plasmids were transfected into the cells with Lipofectamine 2000 according to the manufacturer's instructions, and the transfected cells with no plasmids were set as the control. The coverslips were put into acetone-alcohol solution for 30 min, and then fixed on the slides with glue. Cells were incubated with mouse anti-human monoclonal Ku70 and DNA-PKcs antibodies (NeoMarkers Corporation), immunohistochemistry staining was performed according to the SP kit manual (Beijing Tianwei Time Biotechnology Co. Ltd.). For negative control, primary antibodies were displaced by PBS solutions.

MTT assay. MTT assays were performed to assess the effect of siRNA treatment on cell proliferation. Briefly, $5 \times 10^{3}$ cells/ well were plated on a 96-well plate for $24 \mathrm{~h}$, then Ku70 and DNA-PKcs siRNA plasmids were transfected into the cells by Lipofectamine 2000. After 24, 48, 72 or $96 \mathrm{~h}$ of culture at $37^{\circ} \mathrm{C}, 10 \mu 1$ of MTT $(5 \mathrm{mg} / \mathrm{ml})$ were added to each well. The 
A
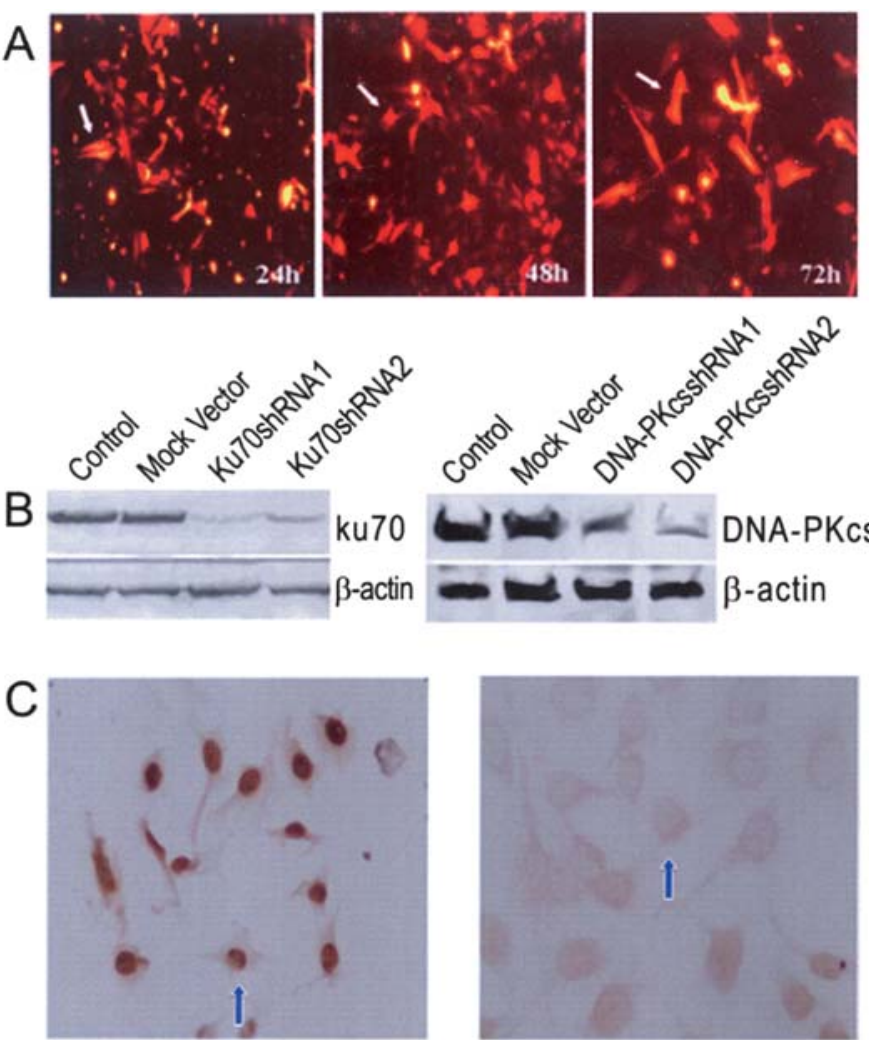

Before transfection, $\mathrm{SP} \times 400$

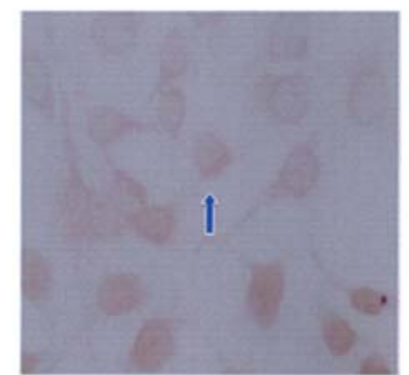

48h after transfection, $\mathrm{SP} \times 400$

Figure 2. (A) Cells were observed under a fluorescent microscope 24, 48 and $72 \mathrm{~h}$ after transfected with recombinant PSIREN-Ku70shRNA1. The highest transfection efficiency was obtained $48 \mathrm{~h}$ after transfection. Similar results were also obtained in the cells transfected with other recombinant plasmids as well as the mock plasmid. (B) In Western blot assay, downregulation of DNA-PKcs and Ku70 protein were observed $48 \mathrm{~h}$ after transfection with recombinant plasmids. (C) In pSIREN-DNAPKcsshRNA2-transfected HeLa cells, DNA-PKcs expression was clearly reduced $48 \mathrm{~h}$ after transfection.

reaction was stopped after $4 \mathrm{~h}$ of incubation by adding $150 \mu \mathrm{l}$ of dimethyl sulfoxide (DMSO). The optical density (OD) value was obtained by measuring absorbance at the wavelength of $570 \mathrm{~nm}$, and the proliferation index calculated by the ratio of OD570 of experimental group to OD570 of control group. The MTT assay was done in triplicate, and repeated 3 times.

FCM. Cells were harvested and fixed in cold $70 \%$ ethanol for $24 \mathrm{~h}$ at $-20^{\circ} \mathrm{C}$, washed with PBS, treated with RNase A for $30 \mathrm{~min}$ at room temperature, and then stained with propidium iodide (PI) for $30 \mathrm{~min}$, PI stained cells were analyzed using a flow cytometer (Becton Dickinson).

Statistical analysis. Statistical analysis was performed using statistical package SPSS12.0. To determine the significant difference (95\% probability) of parameters between sample groups, ANOVA and q-test were utilized.

\section{Results}

Restriction endonuclease analysis of pSIREN-KuTOshRNA and pSIREN-DNA-PKcsshRNA recombinant plasmids. pSIREN-Ku70shRNA and pSIREN-DNA-PKcsshRNA recombinant plasmids were digested by $B a m \mathrm{HI}$ and EcoRI respectively, the acquired bands were consistent with theoretical anticipated 63-bp bands on the agarose gel electrophoresis (Fig. 1B). The sequences were confirmed with no errors by DNA sequencing.

mRNA silence effect of DNA-PKcs and Ku70 detected by RT$P C R$. DNA-PKcs and Ku70 mRNA expression in transfected HeLa cells was detected by RT-PCR at $48 \mathrm{~h}$ after transfection. Compared with the control and mock vector transfected cells, the mRNA expression level of Ku70 and DNA-PKcs in HeLa cells was reduced dramatically after transfection with pSIREN-Ku70shRNA1, 2 or pSIREN-DNA-PKcsshRNA1, 2, indicating the silence effect of the plasmids, and the inhibition effect of pSIREN-Ku70shRNA1 or pSIRENDNA-PKcsshRNA2 was more obvious (Fig. 1C).

Transfection efficiency. pSIREN-DNR-DsRed-Express expressed red fluorescent protein, the transfected cells were observed under a fluorescent microscope. PSIRENKu70shRNA1, 2 and pSIREN-DNA-PKcsshRNA 1, 2 transfected into HeLa cells with high efficiency. Comparing the transfection efficiency of different time points, we found that of $48 \mathrm{~h}$ was the highest (Fig. 2A).

Protein blocking effect of DNA-PKcs and Ku70 detected by Western blotting. The reduction of mRNA was reflected at protein level, as shown by Western blot analysis (Fig. 2B). Recombinant plasmid-transfected HeLa cells, particularly pSIREN-Ku70shRNA1 or pSIREN-DNA-PKcsshRNA2 transfected cells, demonstrated a high inhibition effect of the target genes $48 \mathrm{~h}$ after transfection. The results were matched with those we acquired from RT-PCR.

Cell immunohistochemistry. We verified the down-regulation effect of pSIREN-DNA-PKcsshRNA2 in living cells by immunohistochemistry. We observed that DNA-PKcs expression was concentrated on the cellular nucleus, and was weakened at $48 \mathrm{~h}$ after pSIREN-DNA-PKcsshRNA2 was transfected into HeLa cells (Fig. 2C).

The effect of the recombinant plasmid transfection on cell proliferation. Cell proliferation was clearly inhibited in recombinant plasmid-transfected HeLa cells when compared with untransfected and mock plasmid-transfected cells $(\mathrm{F}=5.022, \mathrm{P}=0.018)$ (Fig. 3A).

HeLa cell proliferation was inhibited when DNA-PKcs and $\mathrm{Ku} 70$ expression was knocked down by RNAi, the cell growth inhibition rate reached as high as 30 and $37 \%$ after pSIREN-Ku70shRNA1 and pSIREN-DNA-PKcsshRNA2 treatment for $72 \mathrm{~h}$ (Fig. 3A). The dose response curve was drawn according to cell inhibition rate and drug concentration logarithm (DDP, VP-16, TPT) at $24 \mathrm{~h}$ after the drugs were added to the cells, which had been transfected with pSIREN-Ku70shRNA1 and pSIREN-DNA-PKcsshRNA2 for $48 \mathrm{~h}$, and obtained $50 \%$ inhibiting concentration $\left(\mathrm{IC}_{50}\right)$ of DDP, VP-16 and TPT (Fig. 3B), the result showed that the sensitivity of HeLa cells to DDP ( $\mathrm{P}=0.001)$, VP-16 $(\mathrm{P}=0.001)$ and TPT $(\mathrm{P}=0.001)$ increased after transfection, and transfection with pSIREN-Ku70shRNA1 was similar with pSIREN-DNA-PKcsshRNA2. 

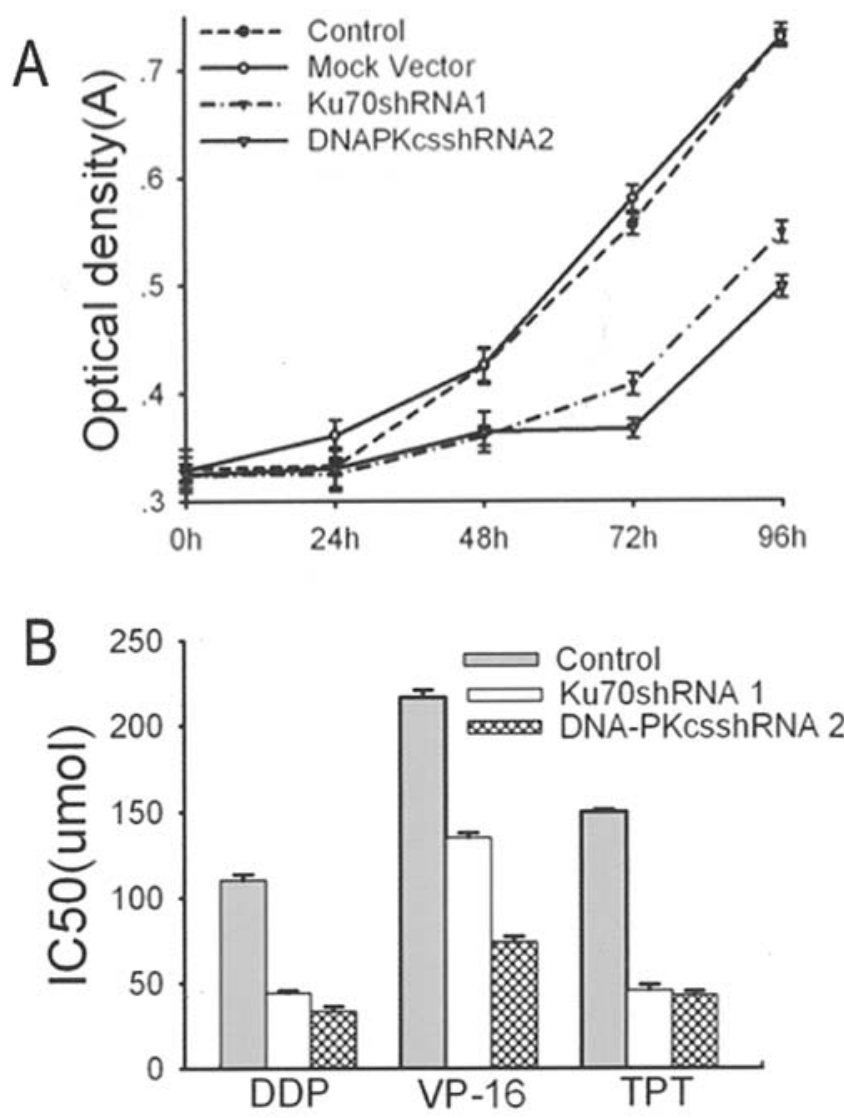

Figure 3. (A) Cell proliferation curves were drawn at different time points after transfection. After transfected with pSIREN-Ku70shRNA1 and pSIREN-DNA-PKcsshRNA2 $72 \mathrm{~h}$ or later, cell proliferation was inhibited when compared with mock plasmid-transfected cells and untransfected cells. (B) After transfection of $48 \mathrm{~h}$ with pSIREN-Ku70shRNA1, pSIREN-DNAPKcsshRNA2 and mock plasmids, HeLa cells were incubated with different concentrations of DDP, VP-16 and TPT. After $24 \mathrm{~h}$, cell proliferation curves were drawn. pSIREN-Ku70shRNA1 and pSIREN-DNA-PKcsshRNA2 transfected cells showed increased sensitivity to drugs compard to mock plasmid-transfected and untransfected cells.

The effects of transfection of the recombinant plasmids on cell cycle and apoptosis. Cell cycle and apoptosis were observed by flow cytometry after $48 \mathrm{~h}$ of pSIRENKu70shRNA1 and pSIREN-DNA-PKcsshRNA2 treatment, the results indicated that the HeLa cell growth was arrested in S phase of the cell cycle following Ku70 and DNA-PKcs knockdown ( $\mathrm{P}=0.001, \mathrm{P}=0.001$; Fig. 4A), apoptotic cells increased $(\mathrm{P}=0.001, \mathrm{P}=0.001$; Fig. 4B). Cell cycle and apoptosis were observed by flow cytometry at $24 \mathrm{~h}$ after 40 $\mu \mathrm{mol} / 1$ of DDP was added to the cells which had been transfected with pSIREN-Ku70shRNA1 and pSIREN-DNAPKcsshRNA2 for $48 \mathrm{~h}$, flow cytometric analysis indicated that apoptosis rates were clearly increased in these cells compared with in that of mock plasmid-transfected cells (Fig. 4B).

\section{Discussion}

DNA-PK is the most important component of mammalian NHEJ, competent DNA-PK participates in the course of transcription and apoptosis via phosphorylation of many
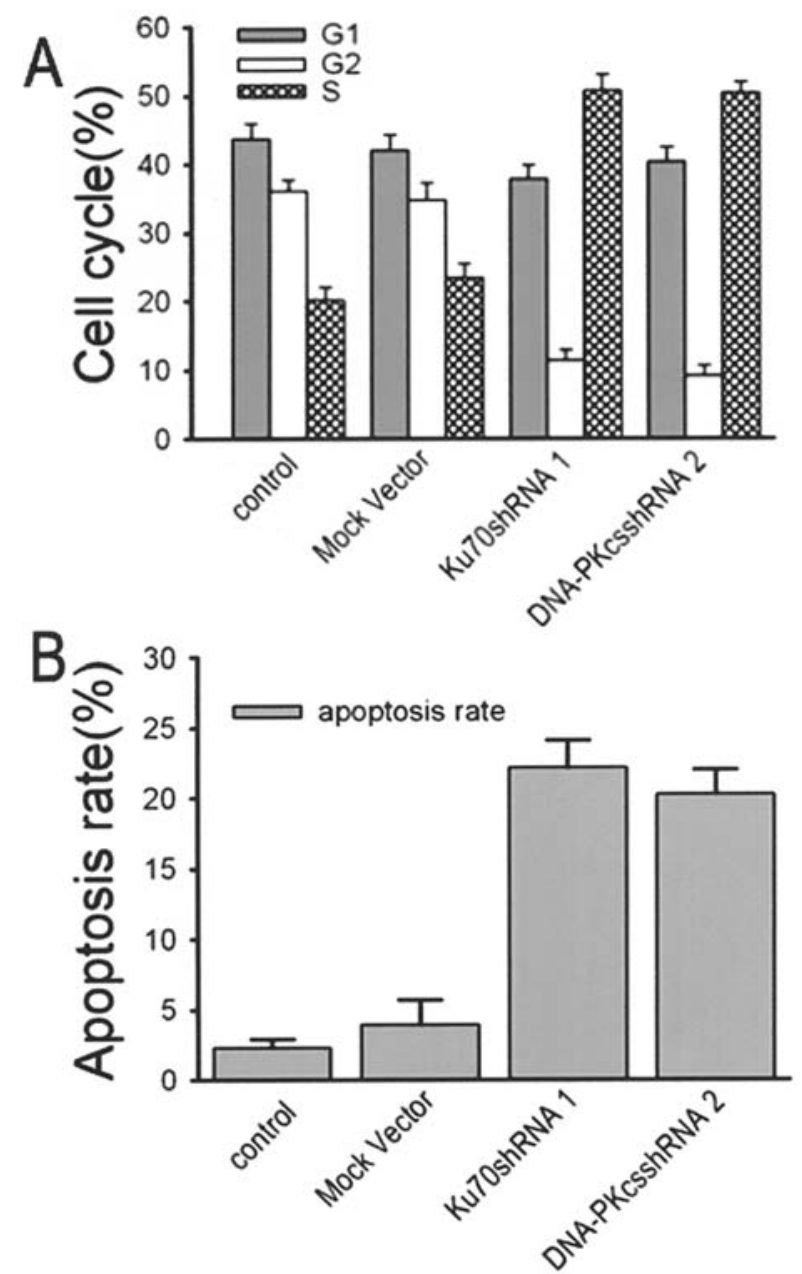

Figure 4. (A) After $48 \mathrm{~h}$ transfection with pSIREN-Ku70shRNA1, pSIRENDNA-PKcsshRNA2 and mock plasmids, HeLa cells were treated for cell cycle analysis by flow cytometry. Most pSIREN-Ku70shRNA1 and pSIREN-DNA-PKcsshRNA2 transfected cells were arrested in S phase. (B) After $48 \mathrm{~h}$ transfection with pSIREN-Ku70shRNA1, pSIREN-DNAPKcsshRNA2 and mock plasmids, HeLa cells were incubated with DDP (40 $\mu \mathrm{mol} / 1)$. after $24 \mathrm{~h}$, cell apoptosis rates were assayed by a flow cytometer. More apoptotic cells were detected in pSIREN-Ku70shRNA1 and pSIREN-DNA-PKcsshRNA2 transfected cells than in the mock plasmid-transfected cells.

protein substrates, and has significance in maintaining the genetic stability (7). Many studies have described that the start of mammalian DNA duplication is Ku-dependent. Ku with activation of ATP enzyme and joining enzyme connected with some duplicate initiation factors, and the absence of $\mathrm{Ku}$ induced a decrease of DNA duplication to an elementary level $(8,9) . \mathrm{Ku}$ interacts with some members of the RecQ family, which belongs to DNA untwisting enzyme and relates to DNA recombination, to participate in liver nucleic acid metabolism (10,11). Interaction between $\mathrm{Ku}$ and Msx2, Runx2, Tbdn 100 regulated the expression of bone Gla protein gene; interaction between $\mathrm{Ku} 70$, which replaces heat shock element (HSE), and heat shock transcription factor 1 (HSF1) inhibited gene promoter of heat shock protein 70 (HSP70) (12). By interactions with human telomerase transcriptase hTERT, Ku regulated telomere longitude and maintained the stability of telomeres (13). DNA-PKcs is 
recognized to function in the pathways of NHEJ of DSBs and $\mathrm{V}(\mathrm{D}) \mathrm{J}$ recombination. In addition, DNA-PKcs is essential for maintaining telomere length and stability, and it can phosphorylate in vitro a number of important proteins including some oncogene products and transduction factors such as c-fos, c-myc, and c-jun. Recent data demonstrated that suppression of the DNA-PK expression enhanced the sensitivity of radio- and chemotherapy in tumor cells, which suggested the potential role of DNA-PK in radio- and chemosensitivity (14-17).

Cisplatin (DDP), etoposide (VP-16) and topotecan (TPT) are widely used clinically for the treatment of tumors. Cisplatin produces a variety of platinum-DNA adducts, including intraand inter-strand crosslinks, and it is generally accepted that these lesions underlie most of the cytotoxic effects of the drug $(18,19)$. The topoisomerase II is a nuclear enzyme that functions during both DNA replication and transcription, topoisomerase II inhibitors, such as VP-16, represent a major class of anticancer agents with documented activities against a broad spectrum of human malignancies (20), can stabilize the complex formed by topoisomerase II and the 5'-cleaved ends of the DNA, thus forming stable (non-repairable) proteinlinked DNA double-strand breaks. Cells are apparently able to recognize such DNA damage and, in turn, eliminate the injured cells by apoptosis (21). Topotecan selectively poisons topoisomerase I by trapping topoisomerase I cleavage complexes, which correspond to enzyme-linked DNA breaks. It induces replication-dependent DNA lesions and arrests cells in the $\mathrm{S}$ and $\mathrm{G} 2$ phase of cell cycle. DNA damage induced by topotecan probably consists of replication-mediated DNA double-strand ends and formation of abnormal replication intermediates, consequent to encounters of replication forks with topotecan-stabilized topoisomerase I-DNA complexes (22).

However, the emergence of tumor cell drug resistance remains a major clinical problem and the frequent cause of failure in the long-term efficacy of cancer therapy $(18,19,23)$. Extensive experimental research has shown that cellular resistance mechanisms are generally multifactorial (24-27). Possible mechanisms of acquired resistance include: a) pretarget events, including drug uptake, metabolism, and intracellular distribution; b) drug-target interactions; and c) post-target events, including macromolecular syntheses, DNA repair, cell cycle progression, and regulation of cell death $(26,28)$.

Because of the reactivity of DDP and DNA topoisomerase inhibitors and the complexity of the cellular response to DNA damage, the molecular mechanisms that underlie chemoresistance are largely unknown. Therefore, the goal of our experiment is to explore the underlying mechanisms responsible for this phenomenon, with the hope that more effective therapies can be devised.

Overexpression of DNA-PK has been reported in a wide variety of human tumors, and many studies suggest that high expression of DNA-PK correlates with radiosensitivity $(17,29)$. Since many antitumor drugs produce a marked effect through damaged DNA in tumor cells, close attention is paid to the relationship of DNA-PK and chemosensitivity. The recombinant plasmids were successfully transfected into HeLa cells and verified by fluorescence microscope (Fig. 2A), the
mRNA and protein expression levels of DNA-PKcs and Ku70 decreased significantly after transfection (Fig. 2), and then showed that DNA-PKcs and Ku70 was knocked down successfully. HeLa cell proliferation was inhibited and the cell cycle arrested in S phase after that Ku70 and DNA-PKcs had been knockdown (Figs. 3A and 4A), while, apoptotic cells increased obviously (Fig. 4B). These findings indicated that DNA-PKcs and $\mathrm{Ku} 70$ are related to cell proliferation and apoptosis. Then, we used DDP, VP-16 and TPT to treat the cells $48 \mathrm{~h}$ after transfection. The $\mathrm{IC}_{50}$ of cells was distinctly decreased compared with the control cells which were treated with drugs alone (Fig. 3B). The cell cycle arrested in S phase in transfected cells (Fig. 4C). The results indicated that the activation of DNA-PK is a critical factor in determining chemosensitivity of tumor cells.

Deriano et al (29) found that at $15 \mathrm{~min}$ after irradiation, the levels of NHEJ (as measured by an in vitro DSB endligation assay) and DNA-PKcs activity were, respectively, 2 -fold and 4-fold higher in radio-resistant than in radiosensitive B-CLL cells; Ku70/Ku80 heterodimer DNA endbinding activity was also 2- to 3-fold higher in the resistant B-CLL cell subset compared with the sensitive B-CLL cell subset. Durant et al (30) inhibited the activity of DNA-PK by Vanillins, a DNA-PK inhibitor, and found it significantly potentiated the cytotoxicity of cisplatin. Eriksson et al (31) inhibited the activity of DNA-PK by trifluoperazine and drew a similar conclusion. We have shown in this study that the inhibition of DNA-PK by mRNA silence of Ku70 or DNAPKcs decreased the activity of DNA-PK and thus induced cell apoptosis. Most importantly, this inhibition correlated with the chemosensitivity of certain drugs, and then DNA-PK possibly participated in the process of drug resistance.

Belenkov et al (28) investigated down-regulation of the Ku 86 gene by transfection of human glioma cell line (M059K) with $200 \mathrm{nmol} / \mathrm{K} \mathrm{Ku} 86$ antisense ASOs which markedly increased cell death after treatment with ionizing radiation, bleomycin, and etoposide; however, no sensitization occurred to the DNA cross-linking agent cisplatin. Ayene et al (32) reported that the expression of Ku70 in HCT116 (colon cancer cell line) had negative correlation with the sensitivity of $\gamma$-ray and VP-16. Frit et al (33) studied a murine CDDPresistant L1210 cell line (L1210/3R) that exhibits crossresistance to IR, and found an increased DNA-end binding activity compared with parental cells (L1210/P). Many studies have described that $\mathrm{Ku}$ is related to radiosensitivity and sensitivity of VP-16, but the relationship between Ku and DDP is not clear. Our findings indicated that inhibition of expression of $\mathrm{Ku} 70$ enhanced the sensitivity of tumor cells to DDP and VP-16. These results suggest that $\mathrm{Ku}$ activity may be an important molecular target in cancer therapy.

Although the studies on the relation between TPT and DNA-PK are few, the studies between TPT and capacity of DNA damage repair have attaracted wide interest $(34,35)$. Our results showed that inhibition activity of DNA-PKcs and Ku70 enhanced the cytotoxicity of TPT.

In conclusion, inhibition of the DNA-PK expression suppressed the growth of tumor cells and enhanced the sensitivity of cells to chemotherapeutic agents DDP, VP-16 and TPT. We provided evidence that DNA-PK may contribute to tumor resistance in chemotherapy. The study strongly 
supported the potential of DNA-PK as a target for reversal of drug resistance.

\section{Acknowledgements}

This study was supported by grants from the National Science Foundation of China $(30025017,30271358,30571950)$ and the '973' Program of China (2002CB513100).

\section{References}

1. Yumoto Y, Shirakawa H, Yoshida M, Suwa A, Watanabe F and Teraoka H: High mobility group proteins 1 and 2 can function as DNA-binding regulatory components for DNA-dependent protein kinase in vitro. J Biochem 124: 519-527, 1998.

2. Pawelczak KS, Andrews BJ and Turchi JJ: Differential activation of DNA-PK based on DNA strand orientation and sequence bias. Nucleic Acids Res 33: 152-161, 2005.

3. Yang J, Yu Y, Hamrick HE and Duerksen-Hughes PJ: ATM, ATR and DNA-PK: initiators of the cellular genotoxic stress responses. Carcinogenesis 24: 1571-1580, 2003.

4. Carney JP, Maser RS, Olivares H, et al: The hMre11/hRad50 protein complex and Nijmegen breakage syndrome: linkage of double-strand break repair to the cellular DNA damage response. Cell 93: 477-486, 1998.

5. Moshous D, Callebaut I, de Chasseval R, et al: Artemis, a novel DNA double-strand break repair/V(D)J recombination protein, is mutated in human severe combined immune deficiency. Cell 105: 177-186, 2001.

6. Nick McElhinny SA, Snowden CM, McCarville J and Ramsden DA: Ku recruits the XRCC4-ligase IV complex to DNA ends. Mol Cell Biol 20: 2996-3003, 2000.

7. Calsou P, Delteil C, Frit P, Drouet J and Salles B: Coordinated assembly of $\mathrm{Ku}$ and p460 subunits of the DNA-dependent protein kinase on DNA ends is necessary for XRCC4-ligase IV recruitment. J Mol Biol 326: 93-103, 2003.

8. Ruiz MT, Nichols A, Price GB and Zannis-Hadjopoulos M: DNA-PKcs-OBA/Ku associate in the absence of DNA, as revealed by two-dimensional capillary gel electromobility shift assay. Electrophoresis 23: 2485-2489, 2002.

9. Matheos D, Ruiz MT, Price GB and Zannis-Hadjopoulos M: Ku antigen, an origin-specific binding protein that associates with replication proteins, is required for mammalian DNA replication. Biochim Biophys Acta 1578: 59-72, 2002.

10. Li B and Comai L: Requirements for the nucleolytic processing of DNA ends by the Werner syndrome protein-Ku70/80 complex. J Biol Chem 276: 9896-9902, 2001.

11. Karmakar P, Snowden CM, Ramsden DA and Bohr VA: Ku heterodimer binds to both ends of the Werner protein and functional interaction occurs at the Werner $\mathrm{N}$-terminus. Nucleic Acids Res 30: 3583-3591, 2002.

12. Tang D, Xie Y, Zhao M, Stevenson MA and Calderwood SK: Repression of the HSP70B promoter by NFIL6, Ku70, and MAPK involves three complementary mechanisms. Biochem Biophys Res Commun 280: 280-285, 2001.

13. Chai W, Ford LP, Lenertz L, Wright WE and Shay JW: Human $\mathrm{Ku} 70 / 80$ associates physically with telomerase through interaction with hTERT. J Biol Chem 277: 47242-47247, 2002.

14. Yazlovitskaya EM and Persons DL: Inhibition of cisplatininduced ATR activity and enhanced sensitivity to cisplatin. Anticancer Res 23: 2275-2279, 2003.

15. Noguchi T, Shibata T, Fumoto S, Uchida Y, Mueller W and Takeno S: DNA-PKcs expression in esophageal cancer as a predictor for chemoradiation therapeutic sensitivity. Ann Surg Oncol 9: 1017-1022, 2002.
16. Eriksson A, Lewensoh R, Larsson R and Nilsson A: DNA dependent protein kinase in leukaemia cells and correlation with drug sensitivity. Anticancer Res 22: 1787-1793, 2002.

17. Omori S, Takiguchi Y, Suda A, et al: Suppression of a DNA double-strand break repair gene, Ku70, increases radio- and chemosensitivity in a human lung carcinoma cell line. DNA Repair 1: 299-310, 2002.

18. Reed JC: Mechanisms of apoptosis avoidance in cancer. Curr Opin Oncol 11: 68-75, 1999.

19. Judson I and Kelland LR: New developments and approaches in the platinum arena. Drugs 59 (Suppl 4): 29-38, 2000.

20. Karpinich NO, Tafani M, Rothman RJ, Russo MA and Farber JL: The course of etoposide-induced apoptosis from damage to DNA and p53 activation to mitochondrial release of cytochrome c J Biol Chem 277: 16547-16552, 2002.

21. Shao RG, Cao CX, Zhang H, Kohn KW, Wold MS and Pommier Y: Replication-mediated DNA damage by camptothecin induces phosphorylation of RPA by DNA-dependent protein kinase and dissociates RPA:DNA-PK complexes. EMBO J 18: 1397-1406, 1999.

22. Andrews PA and Howell SB: Cellular pharmacology of cisplatin: perspectives on mechanisms of acquired resistance. Cancer Cells 2: 35-43, 1990.

23. Beck WT, Morgan SE, Mo YY and Bhat UG: Tumor cell resistance to DNA topoisomerase II inhibitors: new developments. Drug Resist Updat 2: 382-389, 1999.

24. el-Deiry WS: Role of oncogenes in resistance and killing by cancer therapeutic agents. Curr Opin Oncol 9: 79-87, 1997.

25. Larsen AK and Skladanowski A: Cellular resistance to topoisomerase-targeted drugs: from drug uptake to cell death. Biochim Biophys Acta 1400: 257-274, 1998.

26. Pommier Y, Pourquier P, Urasaki Y, Wu J and Laco GS: Topoisomerase I inhibitors: selectivity and cellular resistance. Drug Resist Updat 2: 307-318, 1999

27. Kruczynski A, Barret JM, Van Hille B, et al: Decreased nucleotide excision repair activity and alterations of topoisomerase IIalpha are associated with the in vivo resistance of a P388 leukemia subline to F11782, a novel catalytic inhibitor of topoisomerases I and II. Clin Cancer Res 10: 3156-3168, 2004.

28. Belenkov AI, Paiement JP, Panasci LC, Monia BP and Chow TY: An antisense oligonucleotide targeted to human Ku86 messenger RNA sensitizes M059K malignant glioma cells to ionizing radiation, bleomycin, and etoposide but not DNA cross-linking agents. Cancer Res 62: 5888-5896, 2002.

29. Deriano L, Guipaud O, Merle-Beral H, et al: Human chronic lymphocytic leukemia B cells can escape DNA damage-induced apoptosis through the nonhomologous end-joining DNA repair pathway. Blood 105: 4776-4783, 2005.

30. Durant S and Karran P: Vanillins - a novel family of DNA-PK inhibitors. Nucleic Acids Res 31: 5501-5512, 2003.

31. Eriksson A, Yachnin J, Lewensohn R and Nilsson A: DNAdependent protein kinase is inhibited by trifluoperazine. Biochem Biophys Res Commun 283: 726-731, 2001.

32. Ayene IS, Ford LP and Koch CJ: Ku protein targeting by Ku70 small interfering RNA enhances human cancer cell response to topoisomerase II inhibitor and gamma radiation. Mol Cancer Ther 4: 529-536, 2005.

33. Frit $\mathrm{P}, \mathrm{Canitrot} \mathrm{Y}$, Muller C, et al: Cross-resistance to ionizing radiation in a murine leukemic cell line resistant to cisdichlorodiammineplatinum(II): role of $\mathrm{Ku}$ autoantigen. Mol Pharmacol 56: 141-146, 1999.

34. Kuo CC, Liu JF and Chang JY: DNA repair enzyme, O6methylguanine DNA methyltransferase, modulates cytotoxicity of camptothecin-derived topoisomerase I inhibitors. J Pharmacol Exp Ther 316: 946-954, 2006.

35. Takahashi T, Min Z, Uchida I, et al: Hypersensitivity in DNA mismatch repair-deficient colon carcinoma cells to DNA polymerase reaction inhibitors. Cancer Lett 220: 85-93, 2005. 